\title{
ON SPACES OF MAPS OF $n$-MANIFOLDS INTO THE $n$-SPHERE
}

BY

\author{
VAGN LUNDSGAARD HANSEN
}

\begin{abstract}
The space of (continuous) maps of a closed, oriented manifold $\boldsymbol{C}^{\boldsymbol{n}}$ into the $n$-sphere $S^{n}$ has a countable number of (path-) components. In this paper we make a general study of the homotopy classification problem for such a set of components. For $C^{n}=S^{n}$, the problem was solved in [4], and for an arbitrary closed, oriented surface $C^{2}$, it was solved in [5]. We get a complete solution for manifolds $C^{n}$ of even dimension $n>4$ with vanishing first Betti number. For odd dimensional manifolds $C^{n}$ we show that there are at most two different homotopy types among the components. Finally, for a class of manifolds introduced by Puppe [8] under the name spherelike manifolds, we get a complete analogue to the main theorem in [4] concerning the class of spheres.
\end{abstract}

1. Introduction. Let $C^{n}$ denote a closed, oriented, $n$-dimensional manifold, and let $M\left(C^{n}, S^{n}\right)$ denote the space of (continuous) maps of $C^{n}$ into the $n$-sphere $S^{n}$ equipped with the compact-open topology. According to the Hopf classification theorem, $M\left(C^{n}, S^{n}\right)$ has a countable number of (path-) components enumerated by the degrees of maps of $C^{n}$ into $S^{n}$. Let $M_{k}\left(C^{n}, S^{n}\right)$ denote the component of maps of degree $k$. The purpose of this paper is to make a general study of the homotopy classification problem for the set of components in $M\left(C^{n}, S^{n}\right)$. For $C^{n}=S^{n}$, this problem was solved in [4], and for an arbitrary closed, oriented surface $C^{2}$, it was solved in [5].

For $n$ even we shall prove

THEOREM 1. Let $C^{n}$ be a closed, oriented manifold of even dimension $n \geqslant 4$. Suppose that the first Betti number of $C^{n}$ is zero. Then two components in $M\left(C^{n}, S^{n}\right)$ have the same homotopy type if and only if their associated degrees have the same absolute value.

Theorem 1 is proved in $\$ 4$. It generalizes [4, Theorem 5.1] in even dimensions $n \geqslant 4$ and is parallel to the result in [5] for surfaces.

For $n$ odd we shall prove in $\$ 5$ that there are at most two different homotopy types among the components in $M\left(C^{n}, S^{n}\right)$. This is contained in

THEOREM 2. Let $C^{n}$ be a closed, oriented manifold of odd dimension $n \neq 1,3,7$. Then the component $M_{k}\left(C^{n}, S^{n}\right)$ has the same homotopy type as $M_{0}\left(C^{n}, S^{n}\right)$ if the degree $k$ is even, and as $M_{1}\left(C^{n}, S^{n}\right)$ if the degree $k$ is odd.

Received by the editors April 29, 1980

1980 Mathematics Subject Classification. Primary 58D15; Secondary 55P15.

(C) 1981 American Mathematical Society $0002-9947 / 81 / 0000-0216 / \$ 03.25$ 
If $n=1,3,7$ all the components in $M\left(C^{n}, S^{n}\right)$ trivially have the same homotopy type, since $S^{n}$ in these dimensions has an $H$-space structure, which can be used to construct homotopy equivalences between the various components in $M\left(C^{n}, S^{n}\right)$ by pointwise multiplication of maps. In case $C^{n}=S^{n}$, it was shown in [4] that $M_{0}\left(S^{n}, S^{n}\right)$ and $M_{1}\left(S^{n}, S^{n}\right)$ do not have the same homotopy type for $n \neq 1,3,7$.

For a class of manifolds introduced by Puppe [8] under the name spherelike (sphärenähnliche) manifolds, we can remove the restriction on the Betti number in Theorem 1, and show that $M_{0}\left(C^{n}, S^{n}\right)$ and $M_{1}\left(C^{n}, S^{n}\right)$ do not have the same homotopy type in Theorem 2. The class of spherelike manifolds includes among others spheres and products of spheres and is closed under formation of products and connected sums. We discuss this in $\$ 6$.

The algebraic invariant, which distinguishes between components of different homotopy types, is the $(n-1)$-dimensional homotopy group $\pi_{n-1}\left(M_{k}\left(C^{n}, S^{n}\right)\right)$. In $\S 7$ we present a few concrete calculations of this group up to an extension.

2. Preliminaries. Throughout this paper $C^{n}$ denotes a closed, oriented, topological manifold of dimension $n \geqslant 2$. In fact, a pseudomanifold in the sense of Seifert and Threlfall [9] suffices. All topological spaces will be equipped with a base point, which indiscriminately will be denoted by a * For any pair of based spaces $A$ and $B$, we denote by $\pi(A, B)$ the set of based homotopy classes of based maps of $A$ into $B$. If $A=S^{m}$, the $m$-sphere, we use mostly the standard notation $\pi_{m}(B)=$ $\pi\left(S^{m}, B\right)$. For any based space $X$, we denote by $\Omega^{m} X$ and $\Sigma^{m} X$ respectively the space of $m$-loops on $X$ and the $m$-fold (reduced) suspension of $X$. The symbols $\vee$ and $\wedge$ between based spaces shall denote respectively wedge product and smash product. All mapping spaces will be equipped with the compact-open topology.

For any manifold $C^{n}$ we denote by $M\left(C^{n}, S^{n}\right)$, respectively $F\left(C^{n}, S^{n}\right)$, the space of free maps, respectively the space of based maps, of $C^{n}$ into $S^{n}$. According to the Hopf classification theorem, the components in both $M\left(C^{n}, S^{n}\right)$ and $F\left(C^{n}, S^{n}\right)$ are enumerated by the degrees of maps of $C^{n}$ into $S^{n}$. Let $M_{k}\left(C^{n}, S^{n}\right)$, respectively $F_{k}\left(C^{n}, S^{n}\right)$, denote the component in $M\left(C^{n}, S^{n}\right)$, respectively $F\left(C^{n}, S^{n}\right)$, which consists of the maps of degree $k$. Evaluation at the base point in $C^{n}$ defines a Hurewicz fibration $p_{C, k}: M_{k}\left(C^{n}, S^{n}\right) \rightarrow S^{n}$, which has $F_{k}\left(C^{n}, S^{n}\right)$ as fibre, since $S^{n}$ is simply connected, and therefore a simple space.

Choose once and for all a pair of embedded $n$-discs $D^{n} \subset D_{1}^{n}$ in each closed manifold $C^{n}$, such that the boundaries of these $n$-discs have just the base point in $C^{n}$ in common. Collapsing the boundary $\partial D^{n}$ of $D^{n}$ to the base point defines a map $\nu: C^{n} \rightarrow C^{n} \vee S^{n}$. Let also $\nabla: S^{n} \vee S^{n} \rightarrow S^{n}$ denote the folding map. For any pair of based maps $f: C^{n} \rightarrow S^{n}$ and $g: S^{n} \rightarrow S^{n}$ we can then define the map $f+g: C^{n} \rightarrow S^{n}$ as the composite $\operatorname{map} f+g=\nabla \circ(f \vee g) \circ \nu$.

For each degree $k$ we choose a fixed based map $g_{k}: S^{n} \rightarrow S^{n}$ of degree $k$. Using standard formulas in homotopy theory it is then easy to prove that the map $\theta_{C, k}$ : $F_{0}\left(C^{n}, S^{n}\right) \rightarrow F_{k}\left(C^{n}, S^{n}\right)$, defined by $\theta_{C, k}(f)=f+g_{k}$, is a homotopy equivalence, with an inverse map $\psi_{C, k}: F_{k}\left(C^{n}, S^{n}\right) \rightarrow F_{0}\left(C^{n}, S^{n}\right)$, defined by $\psi_{C, k}(h)=h+g_{-k}$. 
In particular we then get

Proposition 1. For an arbitrary closed, oriented manifold $C^{n}$, all the components in $F\left(C^{n}, S^{n}\right)$ have the same homotopy type.

3. A fundamental diagram. Consider a fixed, closed, oriented manifold $C^{n}$, and let $A=C^{n} \backslash$ Int $D_{1}^{n}$ denote the complement of the interior of the embedded $n$-disc $D_{1}^{n}$ in $C^{n}$. Let $\varphi: S^{n-1} \rightarrow A$ denote an identification map of the $(n-1)$-sphere $S^{n-1}$ with the boundary $\partial D_{1}^{n}$ of $D_{1}^{n}$. Then $C^{n}$ can be identified with the mapping cone for $\varphi$ and we get a mapping sequence

$$
S^{n-1} \stackrel{\varphi}{\rightarrow} A \rightarrow C^{n} \stackrel{q}{\rightarrow} S^{n} \stackrel{\Sigma \varphi}{\rightarrow} \Sigma A \rightarrow \ldots,
$$

where $q: C^{n} \rightarrow S^{n}$ is the map obtained by collapsing $A$ to the base point. Clearly $q$ is a based map of degree 1 .

For each degree $k$, the map $q: C^{n} \rightarrow S^{n}$ of degree 1 induces a map between fibrations by composition of maps,

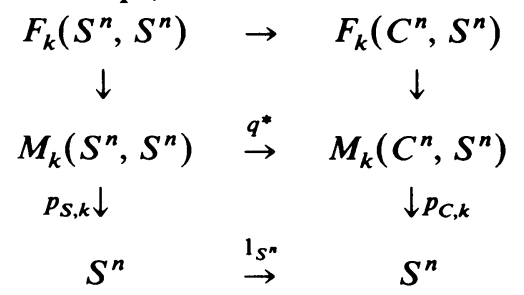

Here $p_{S, k}$ and $p_{C, k}$ are the Hurewicz fibrations defined by evaluation at the base points in respectively $S^{n}$ and $C^{n}$, and $1_{S^{n}}: S^{n} \rightarrow S^{n}$ denotes the identity map on $S^{n}$.

The above map between fibrations induces a map between the associated homotopy sequences:

$$
\begin{aligned}
& \ldots \rightarrow \pi_{n}\left(S^{n}\right) \stackrel{\partial_{S, k}}{\rightarrow} \quad \pi_{n-1}\left(F_{k}\left(S^{n}, S^{n}\right)\right) \quad \rightarrow \quad \pi_{n-1}\left(M_{k}\left(S^{n}, S^{n}\right)\right) \quad \rightarrow 0 \\
& 1 \downarrow \\
& \cdots \quad \rightarrow \pi_{n}\left(S^{n}\right) \quad \underset{\partial_{C, k}}{\rightarrow} \pi_{n-1}\left(F_{k}\left(C^{n}, S^{n}\right)\right) \quad \rightarrow \quad \pi_{n-1}\left(M_{k}\left(C^{n}, S^{n}\right)\right) \quad \rightarrow 0
\end{aligned}
$$

Throughout the paper, we take the constant based maps as base points in $F_{0}\left(S^{n}, S^{n}\right)$ and $F_{0}\left(C^{n}, S^{n}\right)$, and choose the base points in $F_{k}\left(S^{n}, S^{n}\right)$ and $F_{k}\left(C^{n}, S^{n}\right)$, such that the homotopy equivalences $\theta_{S, k}: F_{0}\left(S^{n}, S^{n}\right) \rightarrow F_{k}\left(S^{n}, S^{n}\right)$ and $\theta_{C, k}: F_{0}\left(C^{n}, S^{n}\right) \rightarrow F_{k}\left(C^{n}, S^{n}\right)$ defined in $\S 2$ are base point preserving maps. The addition of based maps, which goes into the definition of $\theta_{C, k}$, is defined using the $n$-disc $D^{n}$, which is suitably contained in the $n$-disc $D_{1}^{n}$ (see $\$ 2$ ), the exterior of which we collapse to obtain $q: C^{n} \rightarrow S^{n}$. We get therefore the following commutative diagram, in which all the vertical maps are induced by $q$ and the unnamed horizontal isomorphisms are the obvious adjoint isomorphisms:

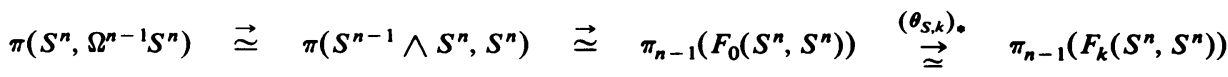

$$
\begin{aligned}
& q^{*} \downarrow \quad \downarrow \quad \downarrow \quad \downarrow
\end{aligned}
$$

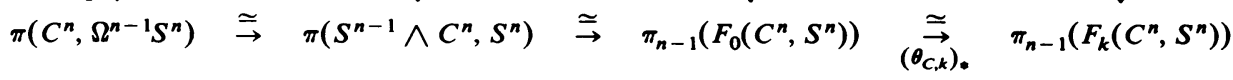


Substituting the information from this commutative diagram into the map between the homotopy sequences for the fibrations $p_{S, k}$ and $p_{C, k}$, without changing the names of the differentials, we get finally the following induced commutative diagram of exact sequences:

$$
\begin{aligned}
& \pi\left(\Sigma A, \Omega^{n-1} S^{n}\right) \\
& \downarrow(\Sigma \varphi)^{*} \\
& \cdots \rightarrow \pi_{n}\left(S^{n}\right) \stackrel{\partial_{S, k}}{\rightarrow} \quad \pi\left(S^{n}, \Omega^{n-1} S^{n}\right) \quad \rightarrow \quad \pi_{n-1}\left(M_{k}\left(S^{n}, S^{n}\right)\right) \quad \rightarrow 0 \\
& \cdots \rightarrow \pi_{n}\left(S^{n}\right) \quad \underset{\partial_{C, k}}{\rightarrow} \pi\left(C^{n}, \Omega^{n-1} S^{n}\right) \quad \rightarrow \quad \pi_{n-1}\left(M_{k}\left(C^{n}, S^{n}\right)\right) \quad \rightarrow 0 \\
& \begin{aligned}
& \downarrow \\
\pi(A, & \left.\Omega^{n-1} S^{n}\right) \\
\downarrow \varphi^{*} &
\end{aligned} \\
& \pi\left(S^{n-1}, \Omega^{n-1} S^{n}\right)
\end{aligned}
$$

The vertical sequence in this diagram is the exact sequence between homotopy groups induced by the mapping sequence:

$$
S^{n-1} \stackrel{\varphi}{\rightarrow} A \rightarrow C^{n} \stackrel{q}{\rightarrow} S^{n} \stackrel{\Sigma \varphi}{\rightarrow} \Sigma A \rightarrow \ldots
$$

The above commutative diagram is the fundamental diagram referred to in the title of this section. The corresponding diagram in dimension 2 was used in [5].

In the following sections we shall freely refer to the homotopy groups and maps in the fundamental diagram.

4. The even dimensional case. Proof of Theorem 1. Throughout this section $C^{n}$ is a closed, oriented manifold of even dimension $n \geqslant 4$. In these dimensions all the homotopy groups in the fundamental diagram are finitely generated abelian groups. For the mapping spaces this follows from Federer [3] or Thom [12].

Let $\iota_{n} \in \pi_{n}\left(S^{n}\right)$ denote the generator represented by the identity map on $S^{n}$. By the delicate suspension theorem, see G. W. Whitehead [14], the Whitehead product

$$
-\left[\iota_{n}, \iota_{n}\right] \in \pi_{2 n-1}\left(S^{n}\right)=\pi\left(S^{n}, \Omega^{n-1} S^{n}\right)
$$

is an element of infinite order. Let $\gamma=q^{*}\left(-\left[\iota_{n}, \iota_{n}\right]\right) \in \pi\left(C^{n}, \Omega^{n-1} S^{n}\right)$.

LEMMA 1. If the first Betti number of $C^{n}$ is zero, then $\gamma$ is an element of infinite order in $\pi\left(C^{n}, \Omega^{n-1} S^{n}\right)$.

Proof. Tensoring the vertical sequence in the fundamental diagram with the rationals $\mathbf{Q}$, we get an exact sequence

$$
\pi\left(\Sigma A, \Omega^{n-1} S^{n}\right) \otimes \mathbf{Q} \stackrel{(\Sigma \varphi)^{*} \otimes 1}{\rightarrow} \pi\left(S^{n}, \Omega^{n-1} S^{n}\right) \otimes \mathbf{Q} \stackrel{q^{*} \otimes 1}{\rightarrow} \pi\left(C^{n}, \Omega^{n-1} S^{n}\right) \otimes \mathbf{Q}
$$

By Serre [10], the rational homotopy type of $\Omega^{n} S^{n}$ is an Eilenberg-Mac Lane space $K(\mathbf{Q}, n-1)$. Using a fundamental result of rationalization for the second 
isomorphism, see e.g. Sullivan [11], we then get

$$
\begin{aligned}
\pi\left(\Sigma A, \Omega^{n-1} S^{n}\right) \otimes \mathbf{Q} & \simeq \pi\left(A, \Omega^{n} S^{n}\right) \otimes \mathbf{Q} \\
& \simeq \pi(A, K(\mathbf{Q}, n-1)) \\
& \simeq H^{n-1}(A ; \mathbf{Q}) .
\end{aligned}
$$

Recall now that $A=C^{n} \backslash$ Int $D_{1}^{n}$, where $D_{1}^{n}$ is an embedded $n$-disc in $C^{n}$. The map $H^{n}\left(C^{n}, A ; \mathbf{Q}\right) \rightarrow H^{n}\left(C^{n} ; \mathbf{Q}\right)$ induced by inclusion is therefore an isomorphism, since $C^{n}$ is oriented; and $H^{n-1}\left(C^{n}, A ; \mathbf{Q}\right)=0$. From the exact cohomology sequence for the pair $\left(C^{n}, A\right)$, we get then immediately the isomorphism

$$
H^{n-1}(A ; \mathbf{Q}) \simeq H^{n-1}\left(C^{n} ; \mathbf{Q}\right) \text {. }
$$

By Poincaré duality and since the first Betti number of $C^{n}$ is zero, we have

$$
H^{n-1}\left(C^{n} ; \mathbf{Q}\right) \simeq H_{1}\left(C^{n} ; \mathbf{Q}\right)=0 .
$$

Putting the above isomorphisms together we get $\pi\left(\Sigma A, \Omega^{n-1} S^{n}\right) \otimes \mathbf{Q}=0$. Consequently,

$$
q^{*} \otimes 1: \pi\left(S^{n}, \Omega^{n-1} S^{n}\right) \otimes \mathbf{Q} \rightarrow \pi\left(C^{n}, \Omega^{n-1} S^{n}\right) \otimes \mathbf{Q}
$$

is a monomorphism.

By Serre [10], $\pi\left(S^{n}, \Omega^{n-1} S^{n}\right) \otimes \mathbf{Q} \simeq \pi_{2 n-1}\left(S^{n}\right) \otimes \mathbf{Q} \simeq \mathbf{Q}$, and the element $-\left[\iota_{n}, \iota_{n}\right] \otimes 1$ is a basis for this rational vector space. Since $q^{*} \otimes 1$ is a monomorphism, the element

$$
\gamma \otimes 1=\left(q^{*} \otimes 1\right)\left(-\left[\iota_{n}, \iota_{n}\right] \otimes 1\right)
$$

generates a 1-dimensional subspace in the rational vector space $\pi\left(C^{n}, \Omega^{n-1} S^{n}\right) \otimes$ Q. Hence $\gamma \in \pi\left(C^{n}, \Omega^{n-1} S^{n}\right)$ must be an element of infinite order. This proves Lemma 1.

The following lemma generalizes [4, Theorem 3.1] in even dimensions $n \geqslant 4$.

Lemma 2. For any degree $k$ put $G_{k}(C)=\pi_{n-1}\left(M_{k}\left(C^{n}, S^{n}\right)\right)$. Then $G_{k}(C)$ is a finitely generated abelian group, and denote by $\operatorname{rank}\left(G_{k}(C)\right)$ and $\operatorname{order}\left(\operatorname{Tor}\left(G_{k}(C)\right)\right)$ respectively the rank of $G_{k}(C)$ and the order of the torsion subgroup of $G_{k}(C)$.

Suppose the first Betti number of $C^{n}$ is zero. Then for each degree $k \neq 0$ we have

(i) $\operatorname{rank}\left(G_{0}(C)\right)=1+\operatorname{rank}\left(G_{k}(C)\right)$.

(ii) $\operatorname{order}\left(\operatorname{Tor}\left(G_{k}(C)\right)\right)=|k| \cdot \operatorname{order}\left(\operatorname{Tor}\left(G_{1}(C)\right)\right)$.

Proof. The proof is inspired by the proof of [4, Theorem 3.1]. We use freely the fundamental diagram from $\$ 3$.

Using a theorem of G. W. Whitehead [13], with a correction for sign by J. H. C. Whitehead [15], it follows that $\partial_{s, k}\left(\iota_{n}\right)=-k\left[\iota_{n}, \iota_{n}\right]$. Hence $\partial_{C, k}\left(\iota_{n}\right)=k \gamma$.

For $k \neq 0$ we put $A_{k}=\partial_{C, k}\left(\pi_{n}\left(S^{n}\right)\right)$. By Lemma $1, A_{k}$ is then an infinite cyclic subgroup of $\pi\left(C^{n}, \Omega^{n-1} S^{n}\right)$ generated by $k \gamma$. Therefore $A_{k}$ is a subgroup of $A_{1}$ with quotient group $A_{1} / A_{k} \simeq \mathbf{Z}_{|k|}$, the cyclic group of order $|k|$.

Still for $k \neq 0$, the triple $A_{k} \subset A_{1} \subset \pi\left(C^{n}, \Omega^{n-1} S^{n}\right)$ induces a short exact sequence

$$
0 \rightarrow A_{1} / A_{k} \rightarrow \pi\left(C^{n}, \Omega^{n-1} S^{n}\right) / A_{k} \rightarrow \pi\left(C^{n}, \Omega^{n-1} S^{n}\right) / A_{1} \rightarrow 0
$$


which is equivalent to the short exact sequence

$$
0 \rightarrow \mathbf{Z}_{|k|} \rightarrow G_{k}(C) \rightarrow G_{1}(C) \rightarrow 0 .
$$

From this sequence we get immediately statement (ii) in Lemma 2.

For $k=0$, observe that the infinite cyclic subgroup in $\pi\left(C^{n}, \Omega^{n-1} S^{n}\right)$ generated by the element $\gamma$ will survive in $G_{0}(C)$, whereas for every $k \neq 0$, it will be reduced to a finite cyclic subgroup in $G_{k}(C)$ by the image of $\partial_{C, k}$. Hence statement (i) in Lemma 2 follows easily. This finishes the proof of Lemma 2.

Theorem 1 stated in the introduction can now easily be proved. First we observe that the components $M_{k}\left(C^{n}, S^{n}\right)$ and $M_{-k}\left(C^{n}, S^{n}\right)$ trivially have the same homotopy type for any degree $k \neq 0$. A homeomorphism $M_{k}\left(C^{n}, S^{n}\right) \rightarrow M_{-k}\left(C^{n}, S^{n}\right)$ can e.g. be constructed by composing the maps in $M_{k}\left(C^{n}, S^{n}\right)$ with a fixed homeomorphism on the $n$-sphere $S^{n}$ of degree -1 . On the other hand it follows by Lemma 2, that two components $M_{k}\left(C^{n}, S^{n}\right)$ and $M_{l}\left(C^{n}, S^{n}\right)$ corresponding to degrees $k$ and $l$ with $|k| \neq|l|$ have nonisomorphic $(n-1)$-dimensional homotopy groups. Hence they have different homotopy types, and Theorem 1 is proved.

5. The odd dimensional case. Proof of Theorem 2. Throughout this section $C^{n}$ is a closed, oriented manifold of odd dimension $n \neq 1,3,7$.

Let $k$ and $l$ be two degrees of maps, and suppose that the fibration $p_{S, k}$ : $M_{k}\left(S^{n}, S^{n}\right) \rightarrow S^{n}$ admits a section $s_{k}: S^{n} \rightarrow M_{k}\left(S^{n}, S^{n}\right)$. Then we can define a map $\Phi$ making the following diagram commutative,

$$
\begin{array}{rlr}
M_{l}\left(C^{n}, S^{n}\right) & \stackrel{\Phi}{\rightarrow} \quad M_{l+k}\left(C^{n}, S^{n}\right) \\
p_{C, l} \searrow & S_{C, l+k}^{n} & \swarrow p_{C, k}
\end{array}
$$

by associating to a map $f: C^{n} \rightarrow S^{n}$ of degree $l$, the map $\Phi(f): C^{n} \rightarrow S^{n}$ of degree $l+k$ obtained by composing the map $\nu: C^{n} \rightarrow C^{n} \vee S^{n}$ defined in $\$ 2$ with the map $C^{n} \vee S^{n} \rightarrow S^{n}$, which restricts to $f$ on $C^{n}$ and to $s_{k}(f(*))$ on $S^{n}$. Observe that $s_{k}(f(*))(*)=f(*)$ so that we get a well-defined map.

The restriction of $\Phi$ to the fibre $F_{l}\left(C^{n}, S^{n}\right)$ of $p_{C, l}$ is a homotopy equivalence

$$
\theta_{C, k, l}: F_{l}\left(C^{n}, S^{n}\right) \rightarrow F_{l+k}\left(C^{n}, S^{n}\right)
$$

similar to the homotopy equivalences $\theta_{C, k}$ defined in $\$ 2$. By a fundamental theorem of Dold [2, Theorem 6.3], $\Phi$ is therefore a fibre homotopy equivalence. In particular, $M_{l}\left(C^{n}, S^{n}\right)$ and $M_{l+k}\left(C^{n}, S^{n}\right)$ have the same homotopy type.

From [4, Lemma 2.2] we know that $p_{S, k}: M_{k}\left(S^{n}, S^{n}\right) \rightarrow S^{n}$ admits a section if and only if the Whitehead product $k\left[\iota_{n}, \iota_{n}\right]=0$. Since $n$ is odd and the Whitehead product is anticommutative, we have $2\left[\iota_{n}, \iota_{n}\right]=0$. Therefore $p_{S, 2}: M_{2}\left(S^{n}, S^{n}\right) \rightarrow S^{n}$ admits a section.

Synthesizing the above analysis it follows that $M_{k}\left(S^{n}, S^{n}\right)$ and $M_{l}\left(S^{n}, S^{n}\right)$ have the same homotopy type if $k \equiv l \bmod 2$. This proves Theorem 2 stated in the introduction.

For odd dimensions $n \neq 1,3,7$, there are therefore at most two different homotopy types among the components in $M\left(C^{n}, S^{n}\right)$. If two homotopy types 
occur, they will be represented by the components $M_{0}\left(C^{n}, S^{n}\right)$ and $M_{1}\left(C^{n}, S^{n}\right)$. We therefore now restrict the investigations to these components and turn again to the fundamental diagram from $\$ 3$.

As a consequence of Adams [1], the Whitehead product $\left[\iota_{n}, \iota_{n}\right] \in \pi_{2 n-1}\left(S^{n}\right) \simeq$ $\pi\left(S^{n}, \Omega^{n-1} S^{n}\right)$ is an element of order 2 in odd dimensions $n \neq 1,3,7$. Using once more the theorem of G. W. Whitehead [13], quoted in the proof of Lemma 2, it follows that $\partial_{C, 1}\left(\iota_{n}\right)=q^{*}\left(\partial_{S, 1}\left(\iota_{n}\right)\right)=q^{*}\left(-\left[\iota_{n}, \iota_{n}\right]\right)=q^{*}\left(\left[\iota_{n}, \iota_{n}\right]\right)$. Consequently, the image of the homomorphism $\partial_{C, 1}$ is a subgroup in $\pi\left(C^{n}, \Omega^{n-1} S^{n}\right)$ of order at most 2 generated by the element $q^{*}\left(\left[\iota_{n}, \iota_{n}\right]\right)$. Let $A_{1}=\partial_{C, 1}\left(\pi_{n}\left(S^{n}\right)\right)$ denote this subgroup.

Since the fibration $p_{C, 0}$ has a section, namely the section of constant maps, the homotopy sequence splits, and we get the first of the isomorphisms

$$
\pi_{n-1}\left(M_{0}\left(C^{n}, S^{n}\right)\right) \simeq \pi_{n-1}\left(F_{0}\left(C^{n}, S^{n}\right)\right) \simeq \pi\left(C^{n}, \Omega^{n-1} S^{n}\right) .
$$

Plugging this information into the homotopy sequence for the fibration $p_{C, 1}$, we obtain the short exact sequence of finitely generated abelian groups

$$
0 \rightarrow A_{1} \rightarrow \pi_{n-1}\left(M_{0}\left(C^{n}, S^{n}\right)\right) \rightarrow \pi_{n-1}\left(M_{1}\left(C^{n}, S^{n}\right)\right) \rightarrow 0 .
$$

If the element $q^{*}\left(\left[\iota_{n}, \iota_{n}\right]\right) \neq 0$, so that $A_{1}$ is a cyclic group of order 2 , this sequence implies in particular the following formula for the orders of the torsion subgroups

$$
\operatorname{order}\left(\operatorname{Tor}\left(\pi_{n-1}\left(M_{0}\left(C^{n}, S^{n}\right)\right)\right)\right)=2 \cdot \operatorname{order}\left(\operatorname{Tor}\left(\pi_{n-1}\left(M_{1}\left(C^{n}, S^{n}\right)\right)\right)\right) \text {. }
$$

As a consequence of this formula we get

Proposition 2. Let $C^{n}$ be a closed, oriented manifold of odd dimension $n \neq 1,3,7$, and suppose that the element $q^{*}\left(\left[\iota_{n}, \iota_{n}\right]\right) \neq 0$ in $\pi\left(C^{n}, \Omega^{n-1} S^{n}\right)$. Then the components $M_{0}\left(C^{n}, S^{n}\right)$ and $M_{1}\left(C^{n}, S^{n}\right)$ have different homotopy types.

In the next section we shall present a class of manifolds for which Proposition 2 can be applied.

6. Spaces of maps of spherelike $n$-manifolds into the $n$-sphere. Let $C^{n}$ be a closed, oriented, topological manifold, or more generally a pseudomanifold in the sense of Seifert and Threlfall [9]. Let $q: C^{n} \rightarrow S^{n}$ be a map of degree 1. For any based space $X$, consider the induced map $q^{*}: \pi\left(S^{n}, X\right) \rightarrow \pi\left(C^{n}, X\right)$. In [8], Puppe studies the question for which manifolds $C^{n}, q^{*}$ is a monomorphism for every $X$. Following Puppe we call a manifold with this property a spherelike (sphärenähnliche) manifold.

Puppe proves that the class of spherelike manifolds includes among others surfaces, spheres and products of spheres, and that it is closed under formation of products and connected sums. For differentiable manifolds $C^{n}$ he proves that $C^{n}$ is spherelike precisely when there exists a closed differentiable submanifold ' $C^{n}$ in euclidean $(n+1)$-space $R^{n+1}$, which can be mapped onto $C^{n}$ by a map of degree 1. He also proves that for a differentiable spherelike manifold $C^{n}$ all the StiefelWhitney classes are trivial.

For a spherelike manifold $C^{n}$, in particular the homomorphism

$$
q^{*}: \pi\left(S^{n}, \Omega^{n-1} S^{n}\right) \rightarrow \pi\left(C^{n}, \Omega^{n-1} S^{n}\right)
$$


is a monomorphism. This implies, that $q^{*}\left(\left[\iota_{n}, \iota_{n}\right]\right)$ is an element of infinite order in $\pi\left(C^{n}, \Omega^{n-1} S^{n}\right)$ for $n$ even, and that $q^{*}\left(\left[\iota_{n}, \iota_{n}\right]\right) \neq 0$ in odd dimensions $n \neq 1,3,7$. By inspection we see therefore, that we can prove Lemma 2 for any spherelike manifold of even dimension $n \geqslant 4$, and that we can apply Proposition 2 in odd dimensions $n \neq 1,3,7$.

Consequently, we get the following theorem, which generalizes the main theorem in [4]. The case $n=2$ is covered by [5], and the cases $n=1,3,7$ are trivial as mentioned in the introduction.

TheOREM 3. Let $C^{n}$ be a closed, oriented, n-dimensional, spherelike manifold. Then the components in $M\left(C^{n}, S^{n}\right)$ are divided into homotopy types as follows.

(i) n even. Two components in $M\left(C^{n}, S^{n}\right)$ have the same homotopy type if and only if their associated degrees have the same absolute value.

(ii) $n$ odd, $\neq 1,3,7$. Two components in $M\left(C^{n}, S^{n}\right)$ have the same homotopy type if and only if their associated degrees have the same parity.

(iii) $n=1,3,7$. All the components in $M\left(C^{n}, S^{n}\right)$ have the same homotopy type.

Theorem 3 supplements Theorem 1 in certain cases, since e.g. products $S^{1} \times$ $S^{n-1}$ for $n$ even are covered by Theorem 3 but not by Theorem 1 .

7. Some calculations of the homotopy group $\pi_{n-1}\left(M_{k}\left(C^{n}, S^{n}\right)\right)$. For certain closed, oriented manifolds $C^{n}$, the homomorphisms $\varphi^{*}$ and $(\Sigma \varphi)^{*}$ in the fundamental diagram from $\$ 3$ are both zero homomorphisms. In such cases, a simple diagram chasing in the fundamental diagram leads to the short exact sequence

$$
0 \rightarrow \pi_{n-1}\left(M_{k}\left(S^{n}, S^{n}\right)\right) \rightarrow \pi_{n-1}\left(M_{k}\left(C^{n}, S^{n}\right)\right) \rightarrow \pi\left(A, \Omega^{n-1} S^{n}\right) \rightarrow 0 .
$$

The group $\pi_{n-1}\left(M_{k}\left(S^{n}, S^{n}\right)\right)$ has been determined for $n=2$ by $\mathrm{Hu}$ [6], and for $n=4,6,8,10,12,14$ by Koh [7]. For such small values of $n$, the above short exact sequence may therefore lead to a calculation of $\pi_{n-1}\left(M_{k}\left(C^{n}, S^{n}\right)\right)$ at least up to an extension.

We shall now present some examples of manifolds $C^{n}$, where the above procedure works. The first example is taken from [5].

EXAMPLE 1. Let $C^{2}=T_{g}$ be a closed, oriented surface of genus $g>0$. Then $A=C^{2} \backslash$ Int $D_{1}^{2}$ has the homotopy type of a wedge of $2 g 1$-spheres, and the homotopy class of $\varphi: S^{1} \rightarrow A$ is a product of commutators. Since $\pi_{1}\left(\Omega^{1} S^{2}\right) \simeq$ $\pi_{2}\left(S^{2}\right)$ and $\pi_{1}\left(\Omega^{2} S^{2}\right) \simeq \pi_{3}\left(S^{2}\right)$ are both abelian groups, a commutator product in either of these groups is trivial, and hence it follows easily that $\varphi^{*}$ and $(\Sigma \varphi)^{*}$ are both zero homomorphisms. We get therefore the short exact sequence

$$
0 \rightarrow \pi_{1}\left(M_{k}\left(S^{2}, S^{2}\right)\right) \rightarrow \pi_{1}\left(M_{k}\left(T_{g}, S^{2}\right)\right) \rightarrow \pi\left(\bigvee_{i=1}^{2 g}\left(S^{1}\right)_{i}, S^{2}\right) \rightarrow 0,
$$

which by substitution of Hu's calculation of $\pi_{1}\left(M_{k}\left(S^{2}, S^{2}\right)\right)$ in [6] leads to the short exact sequence

$$
0 \rightarrow \mathbf{Z}_{2|k|} \rightarrow \pi_{1}\left(M_{k}\left(T_{g}, S^{2}\right)\right) \rightarrow \mathbf{Z}^{2 g} \rightarrow 0 .
$$

Here $\mathbf{Z}_{2|k|}$ denotes the cyclic group of order $2|k|$ (the integers $\mathbf{Z}$ for $k=0$ ), and $\mathbf{Z}^{2 \boldsymbol{g}}$ denotes the free abelian group on $2 g$ generators (the trivial group for $g=0$ ). 
As shown in [5], the extension represented by the above short exact sequence is central.

EXAMPLE 2. Let $C^{n}=S^{r, s}=S^{r} \times S^{s}$, with $n=r+s$ and $r, s>1$, be a product of spheres. In this case $A=C^{n} \backslash$ Int $D_{1}^{n}$ has the homotopy type of the wedge $S^{r} \vee S^{s}$, and $\varphi: S^{n-1} \rightarrow A$ is homotopic to the Whitehead product map $W_{r, s}$ : $S^{r+s-1} \rightarrow S^{r} \vee S^{s}$.

Since Whitehead products vanish in an $H$-space, in particular in $\Omega^{n-1} S^{n}$ and $\Omega^{n} S^{n}$, it is easy to prove that $\varphi^{*}$ and $(\Sigma \varphi)^{*}$ are both zero homomorphisms. We get therefore the short exact sequence

$$
0 \rightarrow \pi_{n-1}\left(M_{k}\left(S^{n}, S^{n}\right)\right) \rightarrow \pi_{n-1}\left(M_{k}\left(S^{r, s}, S^{n}\right)\right) \rightarrow \pi_{r+n-1}\left(S^{n}\right) \oplus \pi_{s+n-1}\left(S^{n}\right) \rightarrow 0 \text {. }
$$

EXAMPLE 3. Let $C^{n}=S_{m}^{r_{i}, s}=\left(S^{r_{1}} \times S^{s_{1}}\right) \# \cdots \#\left(S^{r_{m}} \times S^{s_{m}}\right)$, with $r_{i}+s_{i}=n$ and $r_{i}, s_{i} \geqslant 1$, be a connected sum of products of spheres. In this case $A=$ $C^{n} \backslash$ Int $D_{1}^{n}$ has the homotopy type of the wedge $\bigvee_{i=1}^{m}\left(S^{r_{i}} \bigvee S^{s_{i}}\right)$, and $\varphi: S^{n-1} \rightarrow$ $A$ is homotopic to a sum of Whitehead product maps. Then $\varphi^{*}$ and $(\Sigma \varphi)^{*}$ are again both zero homomorphisms, and we get the short exact sequence

$$
\begin{aligned}
0 & \rightarrow \pi_{n-1}\left(M_{k}\left(S^{n}, S^{n}\right)\right) \rightarrow \pi_{n-1}\left(M_{k}\left(S_{m}^{r_{i}, s_{i}}, S^{n}\right)\right) \\
& \rightarrow \bigoplus_{i=1}^{m}\left[\pi_{r_{i}+n-1}\left(S^{n}\right) \oplus \pi_{s_{i}+n-1}\left(S^{n}\right)\right] \rightarrow 0 .
\end{aligned}
$$

\section{REFERENCES}

1. J. F. Adams, On the non-existence of elements of Hopf invariant one, Ann. of Math. 72 (1960), 20-104.

2. A. Dold, Partitions of unity in the theory of fibrations, Ann. of Math. 78 (1963), 223-255.

3. H. Federer, A study of function spaces by spectral sequences, Trans. Amer. Math. Soc. 82 (1956), 340-361.

4. V. L. Hansen, The homotopy problem for the components in the space of maps on the n-sphere, Quart. J. Math. Oxford Ser. (3) 25 (1974), 313-321.

5. $149-158$. , On the space of maps of a closed surface into the 2-sphere, Math. Scand. 35 (1974),

6. S. T. Hu, Concerning the homotopy groups of the components of the mapping space $Y^{S^{\prime}}$, Indag. Math. 8 (1946), 623-629.

7. S. S. Koh, Note on the properties of the components of the mapping space $X^{S^{p}}$, Proc. Amer. Math. Soc. 11 (1960), 896-904.

8. D. Puppe, Homotopiemengen und ihre induzierten Abbildungen. II, Sphärenähnliche Mannigfaltigkeiten, Math. Z. 69 (1958), 395-417.

9. H. Seifert und W. Threlfall, Lehrbuch der Topologie, Teubner, Leipzig, 1934.

10. J.-P. Serre, Homologie singulière des espaces fibrés. Applications, Ann. of Math. 54 (1951), 425-505.

11. D. Sullivan, Geometric topology. Part I: Localization, periodicity and Galois symmetry, Mimeographed notes, M.I.T., Cambridge, Mass., 1970.

12. R. Thom, L'homologie des espaces functionnels, (Colloque de Topologie Algébrique, Louvain 1956), Thone, Liège; Masson, Paris, 1957, pp. 29-39.

13. G. W. Whitehead, On products in homotopy groups, Ann. of Math. 47 (1946), 460-475.

14. __ A generalization of the Hopf invariant, Ann. of Math. 51 (1950), 192-237.

15. J. H. C. Whitehead, On certain theorems of G. W. Whitehead, Ann. of Math. 58 (1953), 418-428.

Mathematics Institute, The Technical University of Denmark, DK-2800 Lyngby, Denmark 